\title{
EVENTOS ESTRESANTES Y LA RELACIÓN CON EL CONSUMO DE ALCOHOL Y TABACO EN ADOLESCENTES
}

\section{STRESSFUL LIFE EVENTS AND ITS RELATIONSHIP TO ALCOHOL AND TOBACCO CONSUMPTION IN ADOLESCENTS}

\author{
Miguel Ángel Villegas-Pantoja * \\ María Magdalena Alonso-Castillo ${ }^{* *}$ \\ Bertha Alicia Alonso-Castillo ${ }^{* * *}$ \\ Francisco Rafael GuZmán Facundo ${ }^{* * * *}$
}

\begin{abstract}
RESUMEN
Objetivo: Identificar la relación entre la experimentación de eventos estresantes y el consumo de alcohol y tabaco en adolescentes de preparatoria del Área Metropolitana de Monterrey, México. Material y método: Diseño descriptivo correlacional con muestreo probabilístico estratificado $(n=416)$. Se aplicaron los cuestionarios Sucesos de Vida, la Prueba de Identificación de Desórdenes por Uso de Alcohol (AUDIT) y una cédula de datos. Resultados: El número de eventos estresantes correlacionó positivamente con el consumo de bebidas alcohólicas, cigarrillos y puntuación de la Prueba de Identificación de Desórdenes por Uso de Alcohol, particularmente en los dominios de consumo perjudicial y síntomas de dependencia. El consumo de alcohol como de tabaco correlacionaron con mayor intensidad con los eventos estresantes del área de salud. Conclusiones: Vivir más eventos estresantes en la adolescencia se asocia con mayor consumo de alcohol y tabaco, particularmente en términos de consumo excesivo de alcohol. Aunque los eventos estresantes pueden ser factores de riesgo para el consumo de sustancias psicoactivas, también es posible que el consumo de alcohol o tabaco sea fuente de estrés.
\end{abstract}

Palabras clave: Acontecimientos que cambian la vida, trastorno por uso de tabaco, trastornos relacionados con alcohol, adolescente, enfermería psiquiátrica.

\begin{abstract}
Aim: To identify the relationship among experiencing stressful events and alcohol and tobacco consumption in adolescents of vocational school from Metropolitan Area of Monterrey, Mexico. Methods: Descriptive correlational design with a probabilistic and stratified sample $(n=416)$. The instruments Sucesos de Vida, Alcohol Use Disorders Identification Test and a sociodemographic questionnaire were applied. Results: The number of stressful events was positively associated with the consumption of alcoholic beverages, cigarettes and Alcohol Use Disorders Identification Test scores, particularly in the domains of harmful drinking and dependence symptoms. Both alcohol and tobacco use were more strongly correlated with stressful events in the health topic. Conclusions: Living more stressful events in adolescence is associated with increased consumption

\footnotetext{
* Maestro en Ciencias de Enfermería. Becario con subvención del Consejo Nacional de Ciencia y Tecnología (México). Universidad Autónoma de Nuevo León, Monterrey, México.E-mail: miguel_vp@msn.com.

${ }^{* *}$ Doctora en Filosofía con especialidad en Psicología. Profesora Universidad Autónoma de Nuevo León, FAEN. Monterrey, México. E-mail: magdalena_alonso@hotmail.com.

${ }_{* * *}$ Doctora en Bioética, especialista en Patología Clínica y en investigación del fenómeno de drogas. Profesora Universidad Autónoma de Nuevo León, FAEN. Monterrey, México. E-mail: balonso_mx@yahoo.com.mx.

${ }^{* * * *}$ Doctor en Ciencias de Enfermería con especialidad en Psiquiatría. Profesor Universidad Autónoma de Nuevo León, FAEN. Monterrey, México. E-mail: francisco.guzmanf@uanl.mx.
} 
of alcohol and tobacco, particularly in terms of excessive alcohol consumption. Although stressful events may be risk factors for substance use, it's possible that alcohol or tobacco consumption could be a source of stress.

Key words: Life change events, tobacco use disorder, alcohol-related disorders, adolescent, psychiatric nursing.

Fecha recepción: 12/12/12 Fecha aceptación: 09/01/14

\section{INTRODUCCIÓN}

A nivel global, el consumo de sustancias adictivas como el alcohol y tabaco es un grave problema de salud pública debido a las diferentes alteraciones físicas, psicológicas, emocionales y sociales que genera. Sin embargo, algunos grupos etarios, como el de los adolescentes, tienen mayor riesgo de sufrir consecuencias a causa del abuso de estas sustancias (1). La adolescencia es una etapa de desarrollo físico, psicológico y social donde es común que los jóvenes experimenten diversos problemas psicosociales $(2,3)$, los que pueden aumentar el riesgo para la ejecución de conductas no saludables (4). En este sentido, muchos de los usuarios alrededor del mundo experimentan el consumo de alcohol y tabaco por primera vez desde la adolescencia temprana, entre los 10 y 14 años (3), lo cual indica el gran costo que, a futuro, puede padecer el individuo, la sociedad y los sistemas de salud.

El abuso de sustancias psicoactivas es un fenómeno que se asocia con diversos factores biológicos, psicológicos, familiares y sociales que, combinados, podrían incrementar la vulnerabilidad de la experimentación y abuso de drogas (1). En la búsqueda de explicaciones para el inicio del consumo de sustancias, algunos estudios han reportado que ciertas experiencias altamente estresantes se asocian con un mayor consumo de drogas como el alcohol y tabaco en los adolescentes que las han vivido $(5,6)$, particularmente cuando ocurren con elevada frecuencia (7) o en etapas tempranas de la vida (8). De acuer- do a la perspectiva de Holmes y Rahe (9), es posible medir la exposición del estrés a través de los eventos vitales estresantes (EVE), que son acontecimientos que provocan alteraciones en las actividades normales de los individuos debido a la naturaleza o gravedad del evento por sí mismo. Se estima que aunque los diversos EVE varían en su severidad, éstos también pueden ser percibidos e interpretados por el individuo de acuerdo al tipo de experiencia vivida (10-12), de modo que pueden ser considerados por el adolescente como negativos cuando posterior a su ocurrencia implican un perjuicio (por ejemplo, muerte de algún miembro de la familia), o inclusive como positivos cuando después de que acontecen denotan algún beneficio (como el cambiar de institución educativa).

En pocas palabras, de acuerdo a su naturaleza los EVE producirán diferentes respuestas emocionales, cognitivas o conductuales en el individuo, pero también dichas respuestas dependerán de la evaluación cognoscitiva realizada por el sujeto, de sus características personales (particularmente sus estrategias de afrontamiento) y de variables ambientales moduladoras. Los EVE ocurren en diferentes esferas de la vida, como en lo relativo a la familia, las relaciones sociales, a nivel personal, en la escuela o la salud (2), de modo que es posible que ejerzan diferentes efectos sobre la salud del individuo $(10,11,13)$. Sin embargo la experimentación de todo EVE puede afectar el bienestar y salud del adolescente, ya sea directamente sobre el estado emocional o indirectamente, a través de su influencia en las conductas de salud o en la percepción de apoyo social (13). 
Hay evidencia que sugiere que los adolescentes mexicanos se ven expuestos a un gran número de EVE en comparación con adolescentes de otros países (14), lo cual podría representar un riesgo para el inicio del consumo de sustancias adictivas. Estudios recientes en los Estados Unidos (15), China (16), Canadá (17), Chile (13), Islandia (6) y algunas islas del pacífico (18) han documentado asociaciones significativas entre diversos EVE y el consumo de alcohol y tabaco, aunque es común que dichas investigaciones se centren en el estudio de unos pocos EVE o una sustancia psicoactiva. De igual manera, es interesante que el consumo de alcohol se ha evaluado con preguntas en términos de cantidad de bebidas ingeridas o días de ingestión, y no a través de instrumentos como la Prueba de Identificación de Desórdenes por Uso de Alcohol (AUDIT)(19), que identifica a los individuos con patrones de consumo de alcohol excesivo.

A modo de conclusión, los hallazgos no permiten ser generalizados. Aunado a la limitada evidencia localizada, es posible que los estudios realizados en México no reflejen la realidad de las diversas zonas del país debido a las diferencias geográficas, culturales y a las problemáticas sociales. Los hallazgos de la Encuesta Nacional de Adicciones $(20,21)$ indican que el consumo de sustancias por parte de los adolescentes es diferenciado en ciertas regiones del país, de modo que no es posible determinar si los EVE son un problema que en realidad se asocia con el consumo de sustancias en los adolescentes de la región norte del país. Esto principalmente a que sólo se localizaron estudios realizados en el centro de México.

En este sentido, el personal de enfermería podría asumir un papel importante al contribuir a fundamentar estratégicamente un cuidado efectivo para el retraso del consumo de drogas a través del diseño de estrategias de afrontamiento y manejo de los EVE dirigidas a los adolescentes, actividades no realizadas en la actualidad. Con base en lo anterior- mente planteado, se estableció como propósito identificar la relación que existe entre los EVE y el consumo de alcohol y tabaco en adolescentes. Para responder al propósito de la investigación se establecieron los siguientes objetivos específicos:

1. Identificar la relación de los EVE y la cantidad de consumo de alcohol y tabaco.

2. Identificar la relación de los EVE con los dominios de consumo de alcohol del AUDIT.

3. Identificar la relación de los EVE y el consumo de alcohol y tabaco, de acuerdo a las áreas en que se experimentan los EVE (familiar, social, personal, de problemas de conducta, de logros y fracasos, salud y escolar).

\section{MATERIAL Y MÉTODO}

Se eligió un diseño descriptivo correlacional (22). La población consistió en 10.805 adolescentes de seis escuelas preparatorias técnicas del área metropolitana de Monterrey, México. El muestreo fue probabilístico (a través de la función de números aleatorios de Microsoft Excel $\left.{ }^{\circledR}\right)$, estratificado por preparatoria con asignación proporcional al tamaño del estrato. El tamaño de la muestra se calculó mediante el software nQuery Advisor ${ }^{\circledR}$ v4.0 con 95\% de nivel de confianza, límite de error de estimación de 0.05 y tasa de no respuesta del $10 \%$. De esta manera, la muestra final calculada fue de 416 adolescentes.

Con el objetivo de reunir información sobre datos biológicos, sociales y de consumo de alcohol y tabaco se empleó una cédula compuesta por 16 ítemes y una tabla de prevalencia. Por otra parte, para conocer los EVE vividos por los adolescentes se aplicó el cuestionario Sucesos de Vida (23), desarrollado para población adolescente mexicana a base de la escala de Coddington $(10,11)$ y los supuestos de Seiffger-Krenke (12). El cues- 
tionario Sucesos de Vida detecta el riesgo de problemas emocionales mediante la identificación de los EVE experimentados en los últimos 12 meses. Se conforma por una lista de 129 EVE que el adolescente señala de forma dicotómica si los experimentó o no. Los ítemes pertenecen a las áreas familiar, social, personal, de problemas de conducta, de logros y fracasos, salud y escolar.

Los EVE incluidos son de cuatro tipos $y$ poseen un puntaje preestablecido que se suma cuando el participante señala haberlos experimentado: los eventos normativos positivos son sucesos moderadamente estresantes e implican algún beneficio (por ejemplo, empezar a trabajar medio tiempo), suman 1 punto en la escala; los eventos normativos negativos son moderadamente estresantes pero implican algún perjuicio (tener alguna desilusión amorosa), suman 2 puntos; los eventos no normativos positivos son altamente estresantes, poco frecuentes e implican algún beneficio (dejar de consumir alguna droga), suman 3 puntos; finalmente, los eventos no normativos negativos son altamente estresantes, poco frecuentes e implican perjuicio o malestar (sufrir un asalto), suman 5 puntos.

De esta forma, la suma global del cuestionario oscila entre 0 y 464 puntos, donde mayor puntaje indica mayor exposición al estrés $y$, por tanto, mayor posibilidad de alteración del bienestar. El cuestionario se conforma por 28 eventos normativos positivos, 24 normativos negativos, 7 no normativos positivos y 70 no normativos negativos correspondientes a las siete áreas antes mencionadas. Cabe señalar que en esta investigación no se realizó el análisis gráfico de los EVE por área (donde puntuaciones que superan la marca T70 dentro de una escala indican mayor exposición a EVE de lo normal), procedimiento de mayor utilidad para labores de diagnóstico y de análisis cualitativo.

De este instrumento se han reportado índices de confiabilidad KR-20 de 0.89 en población mexicana adolescente (13 a 18 años de edad), escolarizada en preparatorias y secundarias públicas como privadas $(23,24)$, lo cual indica que es un instrumento fiable para la población de adolescentes de México. En el caso de la presente investigación se encontró un índice de confiabilidad KR-20 idéntico al señalado anteriormente.

El segundo instrumento fue la Prueba de Identificación de Desórdenes por Uso de Alcohol (AUDIT) en su versión adaptada para México (25). El AUDIT es un instrumento de cribaje que permite identificar a los individuos con patrones de consumo de alcohol perjudicial. Contiene 10 preguntas de opción múltiple que evalúan tres dominios: consumo de riesgo (reactivos 1 al 3), síntomas de dependencia (reactivos 4 al 6) y consumo perjudicial (reactivos 7 al 10). Las preguntas suman puntos a una escala general que oscila de 0 a 40, donde a mayor puntuación existe mayor implicación con el alcohol. Se considera que puntuaciones totales o mayores a 8 indican un consumo perjudicial o inclusive una posible dependencia al alcohol. Los autores que validaron este instrumento estimaron que el AUDIT posee sensibilidad de $80 \%$ y especificidad de 89\%. Así mismo, hay evidencia (26) que indica que el AUDIT posee alta repetibilidad $(r=0.86)$.

Para realizar esta investigación se contó con la aprobación de las comisiones de Ética e Investigación de la universidad rectora, de las instituciones educativas seleccionadas, así como autorización de los planteles educativos. Cabe señalar que durante el estudio se respetó la libertad de participación del adolescente, se aseguró el anonimato y se protegió la confidencialidad de la información brindada. Posterior a la autorización, en cada una de las preparatorias se solicitaron las listas de alumnos de primero a sexto semestre para realizar la selección aleatoria de los participantes.

A los alumnos seleccionados se les reunió en un aula dispuesta por cada plantel, donde se les notificó los objetivos de la investigación, horarios y se les entregó el 
consentimiento informado y el asentimiento informado (en caso de ser menores de edad). Los adolescentes fueron citados al día siguiente para entregar sus autorizaciones y contestar los cuestionarios. Sólo aquellos que contaron con sus respectivos documentos firmados participaron en el estudio. Después de confirmar su autorización, los participantes fueron instalados en la misma aula y se les entregó un sobre con los cuestionarios auto aplicables en su interior.

Posteriormente se proporcionaron las instrucciones y se procedió al llenado de los instrumentos, mismo que demoró alrededor de 25 minutos. Al término, los adolescentes introdujeron los instrumentos dentro del sobre y éste lo depositaron en una caja ubicada en el aula. En ese momento finalizó su participación y se agradeció su colaboración. Es preciso señalar que de los 416 participantes inicialmente seleccionados e invitados a participar, 33 tuvieron que ser reemplazados probabilísticamente por otros adolescentes debido a que los primeros decidieron no participar o no contaron con autorización de sus padres o tutores. De esta forma se completó la muestra total estimada.
Los datos se analizaron con el paquete SPSS ${ }^{\circledR}$ v20.0 para Mac OSX y se dio respuesta a los objetivos mediante estadística descriptiva e inferencial. Para el análisis de datos de este estudio se empleó la prueba no paramétrica Coeficiente de Correlación de Spearman. La elección de dicha prueba se fundamentó en un análisis de las variables continuas mediante la prueba de Kolmogorov-Smirnov con corrección de Lilliefors, misma que evidenció que los datos no tuvieron distribución normal.

\section{RESULTADOS}

Con relación a las características sociodemográficas de los participantes, existió una proporción similar de hombres $(49.5 \%)$ y mujeres $(50.5 \%)$, de los cuales la mayoría cursaban el primer año de preparatoria técnica (44\%) y se dedicaban sólo a estudiar (87\%). Cabe señalar que las medias de edad de inicio en el consumo de sustancias se ubicaron por debajo de los 15 años (Tabla 1). En lo que concierne al consumo de alcohol, se encon-

Tabla 1. Datos descriptivos de los participantes $(n=416)$.

\begin{tabular}{lcrrrrr}
\hline Variables & $n$ & $\bar{X}$ & Mdn & DE & Min. & Máx. \\
\hline Edad & 416 & 16,4 & 16 & 1,1 & 14 & 21 \\
Edad de inicio (alcohol) & 297 & 14,2 & 15 & 2 & 5 & 20 \\
Edad de inicio (tabaco) & 151 & 14,4 & 15 & 1,9 & 5 & 19 \\
Cantidad de bebidas consumidas (día típico) & 297 & 3,7 & 2 & 3,6 & 1 & 20 \\
Cantidad de cigarrillos consumidos (día típico) & 151 & 1,9 & 1 & 2 & 1 & 15 \\
Número de eventos estresantes & 416 & 28,5 & 20 & 11,7 & 4 & 75 \\
Puntuación de EVE & 416 & 74,5 & 68 & 39,5 & 4 & 271 \\
Puntaje AUDIT & 233 & 5,3 & 4 & 5,5 & 0 & 29 \\
Puntaje de consumo de riesgo & 233 & 3,2 & 3 & 2,6 & 0 & 11 \\
Puntaje de consumo con síntomas de dependencia & 233 & 0,6 & 0 & 1,2 & 0 & 7 \\
Puntaje de consumo perjudicial & 233 & 1,4 & 0 & 2,6 & 0 & 14 \\
\hline
\end{tabular}


tró que el promedio de bebidas alcohólicas consumidas en un día típico fue de 3.7 copas $(M d n=2.0, \mathrm{DE}=3.6)$, lo cual indica un consumo de riesgo. Se considera que cuando hay consumo de riesgo es probable que todavía no se presenten trastornos por el consumo de bebidas alcohólicas, sin embargo existe un aumento del riesgo de consecuencias adversas para el bebedor y los que le rodean (26).

Con relación a las prevalencias de consumo, la sustancia más ingerida alguna vez en la vida y en los últimos siete días fue el alcohol con 71.4\% (IC 95\% [67.0 - 75.7]) y $15.9 \%$ (IC 95\% [8.3 - 19.3]) respectivamente. Para el tabaco, las prevalencias alguna vez en la vida y en los últimos siete días fueron de $36.3 \%$ (IC 95\% [31.6 - 40.9]) y 11.3\% (IC $95 \%$ [2.8 - 14.3]). En lo que concierne a los patrones de consumo de alcohol detectados con el AUDIT, la mayoría de los adolescentes consumidores tuvo un patrón de consumo de riesgo $(73.8 \%$, IC 95\% [68.1 - 79.5]). Por otra parte, el 26.2\% (IC 95\% [20.4 - 31.8]) de los adolescentes consumidores tuvieron un patrón de consumo perjudicial. En lo que respecta a la experimentación de EVE, se identificó que los adolescentes de preparatoria técnica vivieron alrededor de $28.5(\mathrm{DE}=11.7)$ eventos estresantes en el último año, aunque de forma global el número de EVE vividos por hombres $(\bar{X}=28.7, M d n=28.0)$ y mujeres $(\bar{X}=28.3, M d n=27.0)$ fue muy similar. En la Tabla 2 se muestran los primeros diez eventos estresantes con mayor prevalencia en la muestra de estudio.

Tabla 2. Diez eventos estresantes más prevalecientes $(n=416)$.

\begin{tabular}{lllc}
\hline Eventos & $f$ & $\%$ & IC 95\% \\
\hline Mejorar mis relaciones con otras personas & 387 & 93 & $90.5-95.4$ \\
Conocer nuevos amigos & 365 & 87,7 & $84.5-90.9$ \\
Compartir más tiempo con mis amigos o compañeros & 341 & 82 & $78.2-85.6$ \\
Tener más responsabilidades & 330 & 79,3 & $75.4-83.2$ \\
Conocer a alguien que me da buenos consejos & 313 & 75,2 & $71.0-79.4$ \\
Dejar de ver algunos amigos & 311 & 74,8 & $70.5-78.9$ \\
No dormir suficiente & 301 & 72,4 & $78.0-76.6$ \\
Tener un reencuentro con mis amigos & 297 & 71,4 & $67.0-75.7$ \\
Empezar a cambiar físicamente & 287 & 69 & $64.5-73.4$ \\
Empezar la preparatoria & 281 & 67,5 & $63.0-72.0$ \\
\hline
\end{tabular}

A continuación se presenta la respuesta a cada uno de los objetivos planteados. El primer objetivo señaló identificar la relación del puntaje de EVE y la cantidad de consumo de alcohol y tabaco. De acuerdo a esto, se identificó que el puntaje de EVE correlacionó de forma positiva y significativa con el número de bebidas alcohólicas y cigarrillos consumidos en un día típico. Los coeficientes de correlación pueden ser observados en la Tabla 3. En lo que respecta al segundo objetivo -que planteó identificar la relación de los eventos estresantes con los dominios de consumo de alcohol del AUDIT- se encontraron correlaciones positivas y significativas entre la puntuación de EVE con la puntuación global del AUDIT y los puntajes de sus tres dominios. Cabe destacar que los dominios 
Tabla 3. Matriz de Coeficientes de Correlación de Spearman para los eventos estresantes, consumo de alcohol, tabaco y dominios de consumo de alcohol $(n=416)$.

\begin{tabular}{|c|c|c|c|c|c|c|c|}
\hline Variables & 1 & 2 & 3 & 4 & 5 & 6 & 7 \\
\hline 1. Puntaje de EVE & 1 & & & & & & \\
\hline 2. Número de bebidas alcohólicas & $0.226^{\star * *}$ & 1 & & & & & \\
\hline 3. Número de cigarrillos & $0.183^{\star}$ & $0.296^{\star * *}$ & 1 & & & & \\
\hline 4. Puntaje AUDIT & $0.294^{* * *}$ & $0.818^{\star * *}$ & $0.27^{\star \star}$ & 1 & & & \\
\hline 5. Puntaje de consumo de riesgo & $0.201^{\star *}$ & $0.883^{\star * *}$ & $0.282^{\star *}$ & $0.919^{* * *}$ & 1 & & \\
\hline $\begin{array}{l}\text { 6. Puntaje de consumo con } \\
\text { síntomas de dependencia }\end{array}$ & $0.317^{\star \star \star}$ & $0.411^{\star * *}$ & $0.276^{\star \star}$ & $0.604^{\star * *}$ & $0.451^{\star \star \star}$ & 1 & \\
\hline 7. Puntaje de consumo perjudicial & $0.323^{* * *}$ & $0.439^{* * *}$ & $0.186^{*}$ & $0.742^{* * *}$ & 0.508 & $0.456^{\star * *}$ & 1 \\
\hline
\end{tabular}

${ }^{*} p<0.05,{ }^{* *} p<0.01,{ }^{* * *} p<0.001$

de consumo de alcohol "síntomas de dependencia" y "consumo perjudicial" son los que correlacionaron con mayor intensidad con el puntaje de EVE, esto al registrar coeficientes de correlación con un tamaño de efecto moderado.
Finalmente, en la Tabla 4 se muestran los coeficientes que dan respuesta al tercer objetivo, que consistió en identificar la relación del consumo de alcohol y tabaco con el puntaje de EVE de acuerdo a las áreas familiar, social, personal, de problemas de conducta,

Tabla 4. Matriz de Coeficientes de Correlación de Spearman de los eventos estresantes por área y la cantidad de consumo de alcohol y tabaco $(n=416)$.

\begin{tabular}{|c|c|c|c|c|c|c|c|c|c|}
\hline Variables & 1 & 2 & 3 & 4 & 5 & 6 & 7 & 8 & 9 \\
\hline $\begin{array}{l}\text { 1. Número de bebidas } \\
\text { alcohólicas }\end{array}$ & 1 & & & & & & & & \\
\hline 2. Número de cigarrillos & $0.296^{\star * *}$ & 1 & & & & & & & \\
\hline 3. Área personal & $0.160^{\star \star}$ & 0.137 & 1 & & & & & & \\
\hline 4. Área social & $0.184^{* * *}$ & 0.015 & $0.444^{* * *}$ & 1 & & & & & \\
\hline 5. Área escolar & $0.161^{\star *}$ & $0.193^{*}$ & $0.311^{* * *}$ & $0.317^{* * *}$ & 1 & & & & \\
\hline 6. Área de salud & $0.336^{* * *}$ & $0.334^{* * *}$ & $0.398^{* * *}$ & $0.397^{\star * *}$ & $0.364^{* * *}$ & 1 & & & \\
\hline 7. Área de logros y fracasos & $0.117^{\star}$ & 0.134 & $0.483^{* * *}$ & $0.471^{\star * *}$ & $0.331^{\star * *}$ & $0.370^{* * *}$ & 1 & & \\
\hline $\begin{array}{l}\text { 8. Área de problemas de } \\
\text { conducta }\end{array}$ & $0.254^{\star * *}$ & $0.206^{* *}$ & $0.435^{* * *}$ & $0.415^{\star * *}$ & $0.422^{* * *}$ & $0.463^{* * *}$ & $0.406^{\star * *}$ & 1 & \\
\hline 9. Área familiar & 0.066 & 0.064 & $0.484^{* * *}$ & $0.412^{\star * \star}$ & $0.387^{\star * *}$ & $0.311^{\star * *}$ & $0.427^{\star * *}$ & $0.418^{\star \star \star}$ & 1 \\
\hline
\end{tabular}


de logros y fracasos, salud y escolar. En lo que se refiere al número de bebidas alcohólicas consumidas en un día típico, se identificó que correlacionó de forma positiva y con intensidad moderada con los EVE del área de la salud. Se entiende que a mayor puntaje de EVE, mayor consumo de bebidas alcohólicas. También se presentaron correlaciones significativas con los eventos estresantes de las demás áreas, sin embargo éstas se caracterizaron por ser de menor intensidad.

En el caso del número de cigarrillos consumidos en un día típico, éste correlacionó de forma débil y positiva con los EVE del área escolar y de problemas de conducta. De forma similar a lo descrito en el número de bebidas alcohólicas, el número de cigarrillos consumidos en un día típico correlacionó moderadamente con los EVE del área de salud. Cabe señalar que los EVE pertenecientes al área familiar no correlacionaron con el consumo de ninguna sustancia psicoactiva.

\section{DISCUSIÓN Y CONCLUSIÓN}

Los resultados indican que existen relaciones positivas entre la experimentación de EVE por los adolescentes y el consumo de alcohol y tabaco. Las correlaciones positivas encontradas en el primer objetivo muestran que a mayor puntaje de EVE existe un mayor consumo de alcohol y tabaco. Aunque estas relaciones son consideradas como débiles (22), respaldan lo concluido por algunos autores $(6-8,15)$ donde un mayor número de EVE experimentados en etapas tempranas de la vida se asociaron con el consumo tanto de alcohol como tabaco. Es probable que estos hallazgos tengan relación con la forma de afrontar los EVE de manera no saludable durante una etapa como la adolescencia, ya que los jóvenes viven constantes cambios físicos, psicológicos y de conducta, y se estima que probablemente no cuentan con formas más saludables de afrontamiento (1).
En este sentido, Brook et al. (27) encontraron que las conductas internalizadas durante la adolescencia (que son comportamientos problemáticos producidos por el afrontamiento inefectivo de los EVE) predijeron problemas relacionados con el consumo de sustancias como el alcohol. Además, debido a la accesibilidad y la condición de legalidad que poseen los cigarrillos y las bebidas alcohólicas, es posible que los adolescentes recurran al consumo de estas sustancias con el fin de afrontar las situaciones estresantes. Esto debido a que tanto el etanol contenido en las bebidas alcohólicas como el tabaco en los cigarrillos tienen efectos placenteros percibidos como ansiolíticos, y que actúan como reforzadores en el establecimiento de la adicción (28).

En cuanto al segundo objetivo, las relaciones positivas encontradas entre el puntaje de EVE y las puntuaciones de los dominios de consumo de alcohol del AUDIT también indican que a más EVE vividos, más intenso es el consumo de alcohol en los adolescentes. Es preciso señalar que las relaciones entre los EVE y las puntuaciones de las subescalas de síntomas de consumo dependiente y de consumo perjudicial del AUDIT fueron de tipo moderado (22) en comparación con las relaciones débiles encontradas entre los EVE y el consumo de bajo riesgo. Esto indica que posiblemente la vivencia de EVE puede implicar un mayor riesgo para experimentar patrones de consumo de alcohol excesivo, lo cual de alguna forma converge con lo reportado en una revisión bibliográfica (5) donde citan algunas investigaciones que predijeron patrones de intenso consumo de alcohol en breves períodos de tiempo (consumo binge) a través de la vivencia de estresores en la infancia.

Sin embargo, también es posible que los adolescentes que presentan patrones de consumo de alcohol perjudicial o con síntomas de dependencia vivan más EVE en comparación con quienes tienen patrones de consumo de menor riesgo. Por ejemplo, es conocido que 
a medida que aumentan las concentraciones de alcohol en la sangre, disminuye la capacidad asociativa, motora y existe pérdida de reflejos, lo cual pone en riesgo al individuo de provocar accidentes que afecten su integridad y la de las personas que le rodean (29, 30). En este mismo sentido cabe señalar que los efectos negativos producidos por la resaca y la abstinencia alcohólica cursan con importantes niveles de ansiedad, de modo que podrían considerarse como eventos estresantes por sí mismos.

El tercer objetivo permitió conocer las relaciones que existen entre el consumo de alcohol, el consumo de tabaco y los EVE categorizados de acuerdo a los planos de la vida donde éstos se desarrollan. Los hallazgos ponen de manifiesto que el consumo de alcohol correlacionó con más clases de EVE (personal, escolar, logros y fracasos, salud y de problemas de conducta) que el consumo de cigarrillos (área escolar, de salud y de problemas de conducta). Sin embargo, cabe señalar que los EVE relativos al área de salud correlacionaron con mayor intensidad con el consumo de alcohol y tabaco en comparación con los EVE de las demás áreas. Es posible que estas correlaciones también respondan a una falta de recursos para afrontar los EVE, y a que el alcohol -además de poseer efectos farmacológicos- en el contexto social es una sustancia que se emplea como un mecanismo de afrontamiento ante la sensación de ansiedad (31). Quizá resulte más complicado para los adolescentes afrontar de manera saludable los EVE del área de la salud debido a que en muchas ocasiones no cuentan con soluciones posibles ante estos problemas. Esto puede representar un área de oportunidad para el personal de enfermería, puesto que depende del personal de salud provocar cambios en la percepción de los adolescentes sobre sus hábitos de cuidado de la salud y en la manera en que dichos hábitos pueden afectar su salud.

Así mismo, es interesante que los EVE ocurridos en el área familiar no correlacionaron con la ingestión de ninguna sustancia.
Esto es similar a lo encontrado por Booker et al. (15) donde el estrés proveniente de la familia parece tener un efecto protector en los adolescentes al momento de experimentar el uso de cigarrillos. No obstante, en algunas investigaciones se ha encontrado que otros estresores del ámbito familiar, habitualmente con signos de coerción como la violencia familiar, el abuso sexual y el abuso físico, correlacionan de forma positiva con el consumo de sustancias $(6,8)$.

Los resultados encontrados quizá indican que los adolescentes emplean otros métodos para afrontar los EVE, o también que la naturaleza de los EVE integrados en el instrumento corresponden a situaciones específicas que no se relacionan intensamente con el consumo de alcohol y tabaco. En todo caso es necesario realizar más investigación para conocer qué métodos son elegidos por los adolescentes para afrontar este tipo de EVE, pero también consultar otros EVE que los jóvenes consideren relevantes en su vida. Esto ya que es posible que debido a la cultura local perciban de forma diferente los EVE e inclusive vivan diferentes situaciones estresantes no consideradas en el instrumento.

La presente investigación posee algunas limitantes que demandan interpretar los resultados con reserva. El empleo de datos provenientes de un reporte retrospectivo por parte de los adolescentes es una forma aceptada para determinar la exposición a EVE (32), sin embargo, dada la naturaleza transversal del estudio no se pueden realizar inferencias que demuestren causalidad. La experimentación de EVE podría ser un factor de riesgo para el consumo de alcohol y de cigarrillos, pero también el consumir alcohol y tabaco podría involucrar situaciones estresantes o causar ansiedad por su naturaleza farmacológica (28). Por esta razón es preciso realizar investigaciones de tipo longitudinal que permitan complementar que los EVE experimentados causan el consumo de alcohol o tabaco.

Los hallazgos de la presente investigación pueden ser de relevancia para la práctica clí- 
nica y comunitaria, ya que sugieren que en la población adolescente a medida que se experimenta un mayor número de EVE también existe la posibilidad de que aumente el consumo de alcohol o de tabaco. De modo que la medición de EVE a través de cuestionarios autoadministrados puede conjeturar un posible riesgo de abuso de alcohol o tabaco. La ventaja de que el personal de enfermería administre cuestionarios autoaplicados radica en que no se requiere experiencia extensiva en su manejo, es posible aplicarlos en diferentes contextos (consultorios, escuelas, clínicas), se requiere de poco tiempo para completarlos, no representan un costo elevado y se consideran como técnicas no invasivas.

Se recomienda continuar estudiando la relación de los EVE con el uso de sustancias psicoactivas en diferentes poblaciones de adolescentes considerando el sexo, ya que aunque de forma general ambos sexos vivieron un número semejante de EVE, otros autores $(15,16,33)$ han encontrado que las categorías de EVE representan factores de riesgo diferentes para la intención de fumar o beber en hombres y en mujeres. Así mismo, de acuerdo a lo presenciado durante la recolección de datos, sería adecuado incorporar a la escala Sucesos de Vida nuevos EVE que los participantes han referido y que no están descritos. Particularmente aquellos relacionados con la violencia e inseguridad que viven en el contexto donde residen. De esta forma se contribuirá a la validez del instrumento y a una mayor comprensión de los EVE vividos por la población adolescente.

Finalmente, se concluye, en base a los resultados de la presente investigación, que el vivir más eventos vitales estresantes durante la adolescencia se relaciona con un mayor consumo de alcohol o tabaco. Esto se presenta con mayor intensidad en formas de consumo excesivo de bebidas alcohólicas y al vivir eventos estresantes relativos al área de la salud. No obstante, aunque los EVE pueden constituir factores de riesgo para el consumo de drogas, también es posible que el consu- mo de alcohol o tabaco, a su vez, sean fuentes de estrés.

Aunque la naturaleza transversal de este estudio impide identificar el rol de causalidad entre estas variables, los hallazgos invitan a continuar investigando la posibilidad de que la experimentación de EVE constituya una fuente de riesgo para la salud de adolescentes. En este sentido, la generación de conocimiento en este tópico podría traducirse, a futuro, en intervenciones preventivas contra las adicciones. Esto al considerar que los EVE que correlacionaron con mayor intensidad fueron los del área de salud (situaciones relacionadas con los hábitos de higiene personal de los adolescentes), lo cual representa áreas de oportunidad para los profesionales de enfermería en México y Latinoamérica.

\section{REFERENCIAS}

1. Hidalgo MI, Júdez J. Adolescencia de alto riesgo. Consumo de drogas y conductas delictivas. Pediatr Integral. 2007; 11(10): 895-910.

2. Jiménez L, Menéndez S, Hidalgo MV. Un análisis de los acontecimientos vitales estresantes durante la adolescencia. Apuntes de Psicología. 2008; 26(3): 427-40.

3. Fondo de las Naciones Unidas para la Infancia. Estado Mundial de la Infancia 2011. La adolescencia: una época de oportunidades. [Internet]. Nueva York: UNICEF; febrero 2011 [citado 1 octubre 2012]. 148 p. Disponible en: http://www.unicef.org/ spanish/ sowc2011/ pdfs/SOWC-2011Main-Report_SP_02092011.pdf

4. Villatoro JA, Gaytán F, Moreno M, Gutiérrez ML, Oliva N, Bretón M, et al. Tendencias del uso de drogas en la Ciudad de México: encuesta de estudiantes 2009. Salud Mental. 2011; 34(2): 81-94.

5. Enoch MA. The role of early life stress as a predictor for alcohol and drug dependence. Psychopharmacology (Berl). 2011; 214(1): 17-31. 
6. Kristjansson AL, Sigfusdottir ID, Allegrante JP, Helgason AR. Parental divorce and adolescent cigarette smoking and alcohol use: assessing the importance of family conflict. Acta Paediatr. 2009; 98: 537-42.

7. Calvete E, Estévez A. Consumo de drogas en adolescentes: el papel del estrés, la impulsividad y los esquemas relacionados con la falta de límites. Adicciones. 2009; 21(1): 49-56.

8. Rothman EF, Edwards EM, Heeren T, Hingson RW. Adverse childhood experiences predict earlier age of drinking onset: results from a representative US sample of current or former drinkers. Pediatrics. 2008; 122(2): 298-304.

9. Holmes TH, Rahe R. The social readjustment rating scale. J Psychosom Res. 1967; 11:213-18.

10. Coddington RD. The significance of live events as etiologic factors in the diseases of children. I. A survey of professional workers. J Psychosom Res. 1972; 16: 7-18.

11. Coddington RD. The significance of live events as etiologic factors in the diseases of children. II. A study of a normal population. J Psychosom Res. 1972; 16: 205-13. 12. Seiffge-Krenke I. Adolescents' health: A developmental perspective. Chicago: Lawrence Erlbaum Associates Inc.; 1998.

13. Barra E, Cerna R, Kram D, Véliz V. Problemas de salud, estrés, afrontamiento, depresión y apoyo social en adolescentes. Terapia Psicológica. 2006; 24(01): 55-61.

14. Gómez EL, Barcelata B, Durán C, Villafranca L. Sucesos de vida estresantes: un estudio transcultural de adolescentes venezolanos y mexicanos. RIDEP. 2004; 17(1): 123-38.

15. Booker CL, Unger JB, Azen SP, Baezconde-Garbanati L, Lickel B, Johnson CA. A longitudinal analysis of stressful life events, smoking behaviors, and gender differences in a multicultural sample of adolescents. Subst Use Misuse. 2008; 43(11): 1521-43.
16. Booker CL, Unger JB, Azen SP, Baezconde-Garbanati L, Lickel B, Johson CA. Stressful life events and smoking behaviors in Chinese adolescents: A longitudinal analysis. Nicotine Tob Res. 2007; 9(11): 1085-94.

17. Low NCP, Dugas E, O'Loughlin E, Rodríguez D, Contreras G, Chaiton M, et al. Common stressful life events and difficulties are associated with mental health symptoms and substance use in young adolescents. BMC Psychiatry. 2012; 12: 116.

18. Baker CK, Hishinuma ES, Chang JY, Nixon DC. The relationship among exposure to stressful life events, drug use, and violence perpetration in a sample of native Hawaiian, Samoan and Filipino adolescents. J Interpers Violence. 2010; 25(3): 379-99.

19. Saunders JB, Aasland OG, Babor TF, de la Fuente JR, Grant M. Development of the Alcohol Use Disorders Identification Test (AUDIT): WHO Collaborative Project on Early Detection of Persons with Harmful Alcohol Consumption--II. Addiction. 1993 Jun; 88(6): 791-804.

20. Instituto Nacional de Psiquiatría Ramón de la Fuente Muñiz; Instituto Nacional de Salud Pública; Secretaría de Salud. Encuesta Nacional de Adicciones 2011: Reporte de alcohol. [Internet]. México, D.F.: INPRFM; 2012 [citado 21 noviembre 2012]. 92 p. Disponible en: http://portal. salud.gob.mx/sites/salud/descargas/pdf/ ENA_2011_ALCOHOL.pdf

21. Instituto Nacional de Psiquiatría Ramón de la Fuente Muñiz; Instituto Nacional de Salud Pública; Secretaría de Salud. Encuesta Nacional de Adicciones 2011: Reporte de tabaco. [Internet]. México, D.F.: INPRFM; 2012 [citado 21 noviembre 2012]. 89 p. Disponible en: http://portal. salud.gob.mx/sites/salud/descargas/pdf/ ENA_2011_TABACO.pdf

22. Burns N, Grove SK. Investigación en enfermería. 3a ed. Barcelona: Elsevier Espa- 
ña SL; 2004.

23. Gómez-Maqueo EL, León I, Durán C, Bravo E, Velasco E. Los sucesos de vida en dos grupos de adolescentes de diferente nivel socioeconómico. Salud Ment. 2001; 24(05): 17-24.

24. Ávila MR, Heredia MC, Gómez EL, Martínez LV. Confiabilidad interna y estabilidad temporal del cuestionario de sucesos de vida para adolescentes en estudiantes mexicanos. Enseñanza e Investigación en Psicología. 2006; 11(01): 97-113.

25. De la Fuente JR, Kershenobich D. El alcoholismo como problema médico. Rev Fac Med Univ Nac Auton Mex. 1992; 35(2): 47-51.

26. Babor TF, Higgins-Biddle, JC, Saunders JB, Monteiro MG. AUDIT. Cuestionario de Identificación de los trastornos debidos al consumo de alcohol. Pautas para su utilización en atención primaria. [Internet]. Ginebra: OMS; 2001 [citado 1 abril 2014]. 40 p. Disponible en: http:// www.who.int/substance_abuse/activities/en/ AUDITmanualSpanish.pdf

27. Brook JS, Saar NS, Zhang C, Brook DW. Psychosocial antecedents and adverse health consequences related to substance use. Am J Public Health. 2009; 99(3): 563-8.

28. Organización Panamericana de la Salud. Neurodependencia del consumo y de- pendencia de sustancias adictivas. Washington D.C.: OPS; 2004.

29. Ministerio de Sanidad y Consumo. Informe sobre alcohol 2007. [Internet]. Madrid: Ministerio de Sanidad y Consumo, Centro de Publicaciones; febrero 2007 [citado el 12 de septiembre de 2012]. 167 p. Disponible en: http://www.pnsd.msc. es/Categoria2/ publica/pdf/InformeAlcohol.pdf

30. Gruenewald PJ, Johnson FW. Drinking, driving, and crashing: a traffic-flow model of alcohol-related motor vehicle accidents. J Stud Alcohol Drugs. 2010; 71: 237-48.

31. Comeau N, Stewart SH, Loba P. The relations of trait anxiety sensivity, and sensation seeking to adolescents' motivations for alcohol, cigarette, and marijuana use. Addict Behav. 2001; 26: 803-25.

32. Keyes KM, Hatzenbuehler ML, Hasin DS. Stressful life experiences, alcohol consumption, and alcohol use disorders: the epidemiologic evidence for four main types of stressors. Psychopharmacology (Berl). 2011; 218(1): 1-17.

33. Young-Wolff KC, Kendler KS, Ericson $\mathrm{ML}$, Prescott CA. Accounting for the association between childhood maltreatment and alcohol-use disorders in males: a twin study. Psychol Med. 2011; 41: 5970 . 